\title{
Otimização de uma metodologia para análise mineralógica racional de argilominerais
}

\section{(Otimization of rational mineralogical analysis of ceramics)}

\author{
M. L. Varela ${ }^{1}$, R. M. do Nascimento ${ }^{1,2}$, A. E. Martinelli, ${ }^{1,2}$ D. Hotza ${ }^{3}$, D. M. A. Melo' ${ }^{2}$ M. A. F. Melo \\ ${ }^{I}$ Departamento de Engenharia Mecânica, Universidade Federal do Rio Grande do Norte - UFRN \\ C.P. 1524, Campus Lagoa Nova, Natal, RN 59072-970 \\ ${ }^{2}$ Departamento de Ciência e Engenharia de Materiais, Universidade Federal do Rio Grande do Norte - UFRN \\ ${ }^{3}$ Departamento de Ciência e Engenharia de Materiais, Universidade Federal de Santa Catarina - UFSC \\ mvarela@ufrnet.br
}

\begin{abstract}
Resumo
O método proposto para análise mineralógica racional de argilominerais faz uso dos resultados fornecidos pelas análises por difração de raios X (DRX) e fluorescência de raios X (FRX), além de introduzir uma correção no teor de quartzo presente na amostra por meio da curva de quartzo livre levantada para cada tipo de argila analisada. Tais dados servem para montar os sistemas de equações lineares onde cada linha representa um elemento presente em uma das fases detectadas por DRX e cada coluna a fase propriamente dita. A solução desse sistema linear dá-se por meio da sub-rotina $A \cdot X=B$, que fornece como resposta a provável constituição mineralógica, em percentual, dos constituintes da matéria-prima em questão. A junção dessas duas técnicas, análise química e análise racional, possibilita um estudo mais detalhado da matéria-prima a ser usada na produção de massa cerâmica.
\end{abstract}

Palavras-chave: análise racional, argila, argilominerais, quartzo livre.

Abstract

The method proposed herein for the rational mineralogical analysis of ceramics uses conventional results obtained by X-ray diffraction (XRD) and X-ray fluorescence $(X R F)$ in addition to a free quartz content correction evaluated for different types of clays. All this information was collected and used to assemble matrix systems containing linear equations. Each line of the system represents an element present in one of the crystallographic phases detected by XRD, and each column the phase itself. The solution of the equation systems is found from sub-routines such as A.X $=B$, whose output is the likely mineralogical composition of the constituents of a particular clay. The joint use of chemical and rational analyses provides a powerful tool to establish the detailed composition of raw materials used in the formulation of industrial ceramic mixtures.

Keywords: rational mineralogical analysis, clays, clay minerals, free quartz.

\section{INTRODUÇÃO}

A caracterização químico-mineralógica de argilas e a determinação das propriedades que seus componentes atribuem às massas cerâmicas permitem estudar as alterações que devem ser feitas nas massas cerâmicas para melhorar as propriedades de corpos cerâmicos e do produto final [1]. Um dos caminhos que pode ser seguido para o melhor conhecimento das propriedades da matéria-prima é o emprego de duas técnicas, concomitantemente: análise química e análise mineralógica. As análises por difração de raios $\mathrm{X}$ e fluorescência de raios $\mathrm{X}$ fornecem como resultados as fases presentes na argila e a relação dos elementos constituintes da argila com a sua proporção na forma de óxidos, respectivamente. Por meio da combinação da análise mineralógica qualitativa e da análise química quantitativa, onde os elementos são todos considerados existindo na forma de óxidos, têm-se informações suficientes para determinar a composição mineralógica das fases presentes na argila. Essa técnica, conhecida como análise racional, tem como fundamento básico a resolução simultânea de equações lineares montadas para cada fase detectada por difração de raios X [1]. A solução desse sistema linear dá-se por meio da sub-rotina A.X $=\mathrm{B}$, que fornece como resposta a provável constituição mineralógica dos constituintes da matéria-prima em questão. Com isso pode ser definida a necessidade ou não da correção da massa e ainda se esta correção é viável ou não, economizando-se, desta forma, tempo e investimento financeiro.

Existem três procedimentos difundidos para a realização dos cálculos de análise mineralógica racional de argilominerais, a saber: o procedimento convencional [2], o procedimento utilizando o método IRTEC [3] e o terceiro procedimento utilizando o método UFSC [1], que surgiu baseado no método IRTEC. Nenhum desses métodos de análise mineralógica racional de argilominerais leva em 
consideração o possível teor de quartzo livre presente na amostra. Estes métodos consideram que o percentual $\mathrm{de}_{\mathrm{SiO}}$ resultante da análise química está combinado com outros elementos formando outras fases, como feldspato, mica e outros. Dessa forma, a presença de quartzo livre, não contabilizado, na matéria-prima pode causar um comportamento distinto da massa cerâmica e, conseqüentemente, um produto final diferente do esperado. Um quarto método é apresentado neste artigo, que aqui será denominado de método "MIDS", que além das considerações usuais da análise racional introduz em seu algoritmo a curva de quartzo livre da matéria-prima analisada.

\section{Método MIDS}

O método MIDS parte dos mesmos princípios propostos pelo método UFSC, ou seja, a resolução simultânea de equações lineares montadas a partir dos resultados fornecidos pelas análises química quantitativa e mineralógica qualitativa. A resolução desse sistema linear é via mínimos quadrados nãonegativos [4], para manter as restrições propostas pelo método UFSC, ou seja, além da necessidade da soma das fases ser igual a 100, que todos os valores resultantes devam ser positivos. Diferentemente do método UFSC, o método MIDS introduz em seu procedimento de cálculo os resultados fornecidos pela curva de calibração de quartzo livre. $\mathrm{O}$ valor fornecido pela curva de quartzo livre é parte integrante do sistema de equações que determina a quantificação das fases existente na amostra. Assim, o número de equações do sistema é definido pelo número de componentes considerado usuais na argila. Esses componentes são aqueles que estão presentes em pelo menos um mineral detectado por difração de raios X. Neste método, para cada componente existente na argila é construída uma equação linear, além da equação referente ao percentual de quartzo livre. Desse modo, a soma das fases mineralógicas dos componentes é proporcional ao somatório total encontrado na análise química. A parte que falta para se completar os $100 \%$ do somatório total é denominada "acessórios", onde estão incluídos todos os componentes que por algum motivo não foram identificados em nenhuma das análises mencionadas.

Assim, para um constituinte $\mathrm{m}$ com $\mathrm{n}$ fases mineralógicas presentes, um sistema com $m+1$ equações é montado a partir de:

$$
\begin{aligned}
& \mathrm{A}_{11} \mathrm{X}_{1}+\mathrm{A}_{12} \mathrm{X}_{2}+\ldots+\mathrm{A}_{1 \mathrm{n}} \mathrm{X}_{\mathrm{n}}=\mathrm{B}_{1} \\
& \mathrm{~A}_{21} \mathrm{X}_{1}+\mathrm{A}_{22} \mathrm{X}_{2}+\ldots+\mathrm{A}_{2 \mathrm{n}} \mathrm{X}_{\mathrm{n}}=\mathrm{B}_{2} \\
& \ldots \\
& \mathrm{A}_{\mathrm{m} 1} \mathrm{X}_{1}+\mathrm{A}_{\mathrm{m} 2} \mathrm{X}_{2}+\ldots+\mathrm{A}_{\mathrm{mn}}+1 \mathrm{X}_{\mathrm{n}+1}=\mathrm{B}_{\mathrm{m}+1}
\end{aligned}
$$

O sistema está sujeito a algumas restrições, a saber:

$$
\mathrm{X}_{1}+\mathrm{X}_{2}+\ldots \mathrm{X}_{\mathrm{n}+1}=100
$$

Com

$$
X_{j}>0, \operatorname{com} j=1 \ldots n, i=1 \ldots m+1
$$

na qual $\mathrm{X}_{\mathrm{j}}=\mathrm{o}$ total dos minerais a ser calculado; $\mathrm{A}_{\mathrm{ij}}=\mathrm{o}$ total dos elementos i no mineral $\mathrm{j}$ da composição teórica deste mineral; $\mathrm{B}_{\mathrm{i}}=\mathrm{o}$ total do elemento $\mathrm{i}$ da análise química da amostra.

\section{MATERIAIS E MÉTODOS}

\section{Determinação da curva de quartzo livre}

Para a realização do levantamento da curva de quartzo livre utilizaram-se padrões de quartzo e caulinita. Estes foram submetidos a moagem e passados em peneira ABNT \#200 $(75 \mu \mathrm{m})$. As frações passantes foram caracterizadas quanto à composição mineralógica por difração de raios $\mathrm{X}$ com o equipamento Shimadzu XRD 6000. As amostras foram submetidas às seguintes condições de análise: radiação $\mathrm{K} \alpha$ de um anodo de cobre $(\lambda=1,54056 \AA), 40 \mathrm{kV}$ e $30 \mathrm{~mA}$. As condições foram: velocidade $1,5 \mathrm{grau} / \mathrm{min}$, faixa de varredura (20) de $5^{\circ}$ a $70^{\circ}$, passo de varredura $0,02^{\circ}$ e tempo por passo $1 \mathrm{~s}$. A identificação das fases foi realizada comparando-se os difratogramas obtidos com arquivos JCPDS.

A caulinita (39,5\% de $\mathrm{Al}_{2} \mathrm{O}_{3}, 46,5 \%$ de $\mathrm{SiO}_{2}$ e $14,0 \%$ de $\mathrm{H}_{2} \mathrm{O}$ ) foi escolhida para a determinação da curva de quartzo livre por ser um argilomineral de grande importância na produção de cerâmica e por possuir em sua fórmula teórica $\mathrm{SiO}_{2}$, que aparecerá somado ao quartzo livre na análise química, tornando o trabalho mais completo. Além de estar presente em praticamente todas as matérias-primas utilizadas na fabricação de produtos cerâmicos, seja em grande ou pequena proporção.

A metodologia utilizada para a preparação das amostras constou da dopagem da caulinita por teores do quartzo padrão que variaram de $5,10,15$ e $25 \%$ em massa. Após a dopagem, o material foi homogeneizado em almofariz durante $5 \mathrm{~min}$. Com as amostras devidamente homogeneizadas, foram preparadas lâminas por prensagem a seco [5]. As lâminas foram submetidas à análise por DRX nas mesmas condições supracitadas. Para a determinação da curva de quartzo livre, utilizou-se a relação da intensidade do pico de quartzo da amostra $\left(I_{i}\right)$ no plano pré-determinado (101) e a intensidade do pico do padrão de quartzo adotado $\left(\mathrm{I}_{\text {ref }}\right)$ no mesmo plano (101). Esta relação $\left(\mathrm{I}_{i} / \mathrm{I}_{\text {ref }}\right)$ possibilita determinar as constantes (K) e (C) que são características de cada instrumento [6]. Após o ajuste da curva, essas constantes $(\mathrm{K})$ e (C) são, respectivamente, os coeficientes angular e linear da curva e permitem interpolações para os valores de quartzo livre desejados. A equação (D) é utilizada para a determinação do percentual de quartzo livre (Xi):

$$
\mathrm{X}_{\mathrm{i}}=\left(\mathrm{I}_{\mathrm{i}} / \mathrm{I}_{\mathrm{ref}}+\mathrm{C}\right) \cdot \mathrm{K}
$$

na qual $X_{i}=$ percentual de quartzo livre que se deseja determinar; $\mathrm{I}_{\mathrm{i}}=$ intensidade do pico de quartzo da amostra; $\mathrm{I}_{\text {ref }}=$ intensidade do pico de quartzo do padrão adotado; $K^{\prime}=1 /$ Coeficiente angular da curva $(\mathrm{K}) ; \mathrm{C}=$ Coeficiente linear da curva. 


\section{Padrões utilizados}

Foi utilizado como padrão de quartzo um pegmatito natural caracterizado por DRX. Obteve-se como resultado o valor da intensidade do pico de quartzo $\left(\mathrm{I}_{\text {ref }}\right)$, que é utilizado como referência para a determinação da curva de quartzo livre (Fig. 1).

A caulinita utilizada como padrão foi adquirida comercialmente e é utilizada como padrão na calibração do DRX. Sua análise apresentou como resultado um material de elevada pureza (Fig. 2).

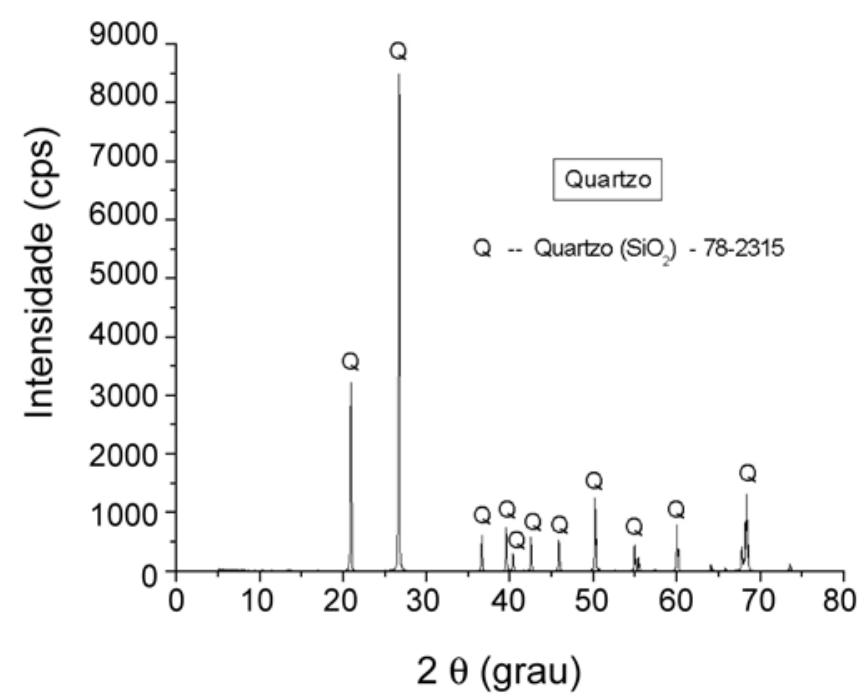

Figura 1: Difratograma de raios $\mathrm{X}$ do padrão de quartzo adotado $\left(\mathrm{I}_{\text {ref }}=8498\right)$.

[Figure 1: X-ray diffraction pattern of standard quartz.]

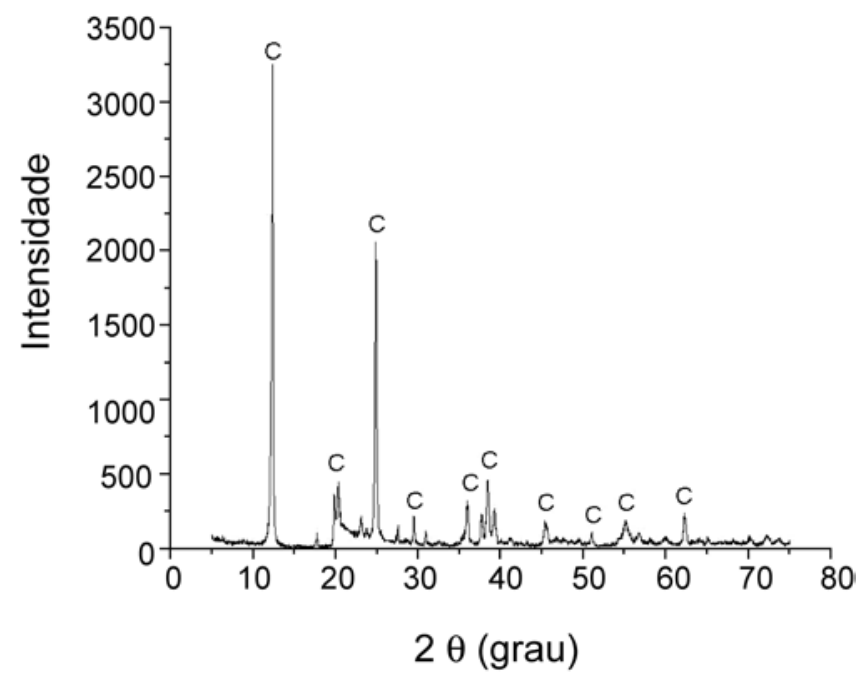

Figura 2: Difratograma de raios $\mathrm{X}$ do padrão de caulinita (C). [Figure 2: X-Ray diffraction pattern of standard kaolinite $(C)$.]

Cálculo das fases mineralógicas

Para o cálculo de cada fase mineralógica deve-se expressar a fórmula teórica de cada fase mineralógica identificada na análise qualitativa em forma de óxidos. De posse dessa informação, calcula-se a fração em massa para cada óxido por meio da sua massa atômica, posteriormente deve-se calcular a fração em massa para cada elemento pertencente ao óxido, também por meio da sua massa atômica. Após esse procedimento, constrói-se uma matriz "A", onde as linhas correspondem ao total de cada elemento pertencente àquela fase, exceto a última que representa a soma de cada fase da amostra e as colunas correspondem a cada fase que se deseja calcular na amostra exceto a última que representa o total de cada elemento da amostra. O quartzo livre obtido na curva de referência é introduzido como uma equação a mais no sistema, inserindo desta forma mais uma fase pertencente a amostra que deverá ser analisada. $\mathrm{O}$ total requisitado pela forma teórica pode ser menor que o determinado pela análise química; nesse caso, é adicionada a esse total uma fase hipotética, denominada "acessórios" para completar 100\%.

Para solução do sistema montado pelos vários componentes da argila, usa-se a sub-rotina $A . X=B$, seguindo o algoritmo descrito por Lawson [4]. Para a validação do método apresentado, foram tomadas matérias-primas puras, Tabela I, e realizada uma mistura de $60 \%$ de caulinita, $25 \%$ de ortoclásio, $10 \%$ de talco e $5 \%$ de acessórios. Com a quantidade de cada matéria-prima na mistura e suas fórmulas teóricas, serão realizadas as análises químicas e, conseqüentemente, a quantificação via método MIDS. Os resultados obtidos serão comparados e analisados em relação aos originais identificados como padrão, e apresentados a seguir.

Tabela I - Fases cerâmicas puras usadas como matériasprimas hipotéticas.

[Table I-Pure ceramic phases used as hypothetical raw material.]

\begin{tabular}{cc}
\hline Matérias-primas & Fórmula teórica \\
\hline Caulinita & $\mathrm{Al}_{2} \mathrm{O}_{3} \cdot 2 \mathrm{SiO}_{2} \cdot 2 \mathrm{H}_{2} \mathrm{O}$ \\
Ilita & $3 \mathrm{~K}_{2} \mathrm{O} \cdot 11 \mathrm{Al}_{2} \mathrm{O}_{3} \cdot 26 \mathrm{SiO}_{2} \cdot 8 \mathrm{H}_{2} \mathrm{O}$ \\
Ortoclásio & $\mathrm{K}_{2} \mathrm{O} \cdot \mathrm{Al}_{2} \mathrm{O}_{3} \cdot 6 \mathrm{SiO}_{2}$ \\
Quartzo & $\mathrm{SiO}_{2}$ \\
Talco & $\mathrm{MgO} \cdot 4 \mathrm{SiO}_{2} \cdot \mathrm{H}_{2} \mathrm{O}$ \\
\hline
\end{tabular}

\section{RESULTADOS E DISCUSSÃO}

Os resultados obtidos serão agora apresentados e comparados com os padrões utilizados para validação da metodologia proposta. Na Tabela II é apresentado o resultado obtido na análise por FRX, na forma de óxido, para a mistura padrão descrita anteriormente.

$\mathrm{Na}$ Tabela III é apresentada a matriz A, do algoritmo $A . X=B$, montada a partir dos resultados obtidos na análise por difração de raios $X$, ou seja, com as fases detectadas nesta análise e os respectivos percentuais de óxido presente em cada fases. 
Tabela II - Análise química do padrão utilizado. [Table II: Chemical analysis of used standard.]

\begin{tabular}{cc}
\hline & Mistura Padrão \\
\hline $\mathrm{SiO}_{2}$ & 50,46 \\
$\mathrm{Al}_{2} \mathrm{O}_{3}$ & 28,28 \\
$\mathrm{~K}_{2} \mathrm{O}$ & 4,71 \\
$\mathrm{MgO}$ & 3,19 \\
\hline
\end{tabular}

Tabela III - Matriz "A" da mistura. [Table III - Mixture matrix " $A$ “.]

\begin{tabular}{lccccc}
\hline & Caulinita & Ortoclásio & Talco & $\begin{array}{c}\text { Quartzo Acessórios } \\
\text { Livre }\end{array}$ \\
\hline $\mathrm{SiO}_{2}$ & 0,218 & 0,303 & 0,296 & 0 & 0 \\
$\mathrm{Al}_{2} \mathrm{O}_{3}$ & 0,209 & 0,097 & 0 & 0 & 0 \\
$\mathrm{~K}_{2} \mathrm{O}$ & 0 & 0,145 & 0 & 0 & 0 \\
$\mathrm{MgO}$ & 0 & 0 & 0,192 & 0 & 0 \\
$\mathrm{SiO}_{2}$ livre & 0 & 0 & 0 & 0,467 & 0 \\
Somatório & 1 & 1 & 1 & 1 & 1 \\
\hline
\end{tabular}

A Tabela IV apresenta a Matriz B, do algoritmo $A \cdot X=B$, com os resultados obtidos na análise por FRX, transformado no percentual da fração mássica de cada elemento nos óxidos.

\begin{tabular}{|c|c|}
\hline \multicolumn{2}{|c|}{$\begin{array}{c}\text { \% da fração mássica } \\
\text { de cada elemento dos óxidos }\end{array}$} \\
\hline $\mathrm{SiO}_{2}$ & 23,59 \\
\hline $\mathrm{Al}_{2} \mathrm{O}_{3}^{2}$ & 14,97 \\
\hline $\mathrm{K}_{2}^{2} \mathrm{O}^{3}$ & 3,91 \\
\hline $\mathrm{MgO}$ & 1,92 \\
\hline $\mathrm{SiO}_{2}$ livre & 0,95 \\
\hline Somatório & 100 \\
\hline
\end{tabular}

Com as matrizes A e B montadas, entra-se na curva de calibração de quartzo livre previamente determinada, com o valor da razão entre a intensidade do pico de quartzo da amostra e a intensidade do pico de quartzo do padrão adotado $\left(\mathrm{I}_{\mathrm{i}} / \mathrm{I}_{\mathrm{ref}}\right)$ em um plano $(h k l)$ pré-determinado e obtém-se o provável percentual de quartzo livre existente na matériaprima, como indicado na Fig. 3.

De posse de todos os dados necessários para a realização dos cálculos, procede-se com a execução do algoritmo $A . X=B=>$ $X=B . A^{-1}$ e tem-se o valor de $X$, ou seja, o valor dos percentuais das fases presentes na mistura. A Tabela $\mathrm{V}$ mostra o resultado obtido através do método MIDS e o padrão utilizado.

$\mathrm{O}$ erro relativo do método apresentado foi calculado

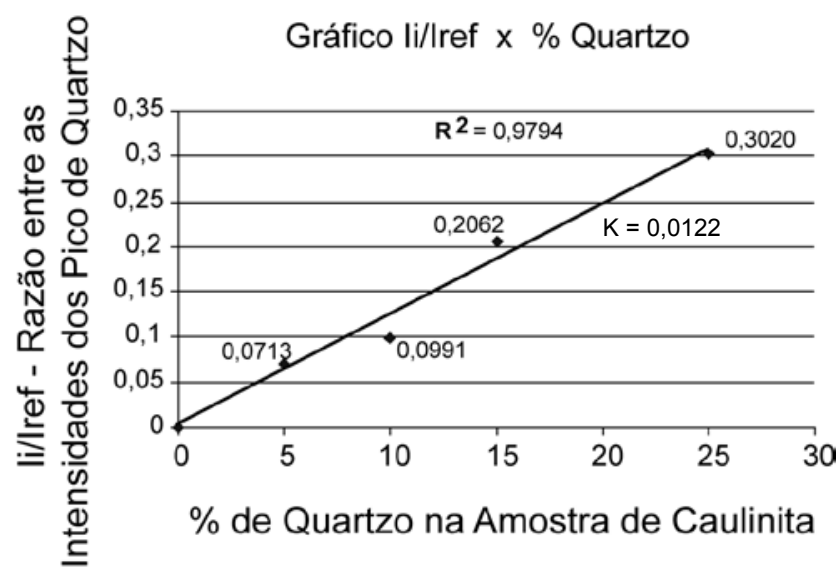

Figura 3: Curva de calibração de quartzo livre da caulinita. [Figure 3: Calibration curve for free quartz in kaolinite.]

Tabela V - Composições padrões e composições calculadas pelo método MIDS.

[Table V - Standard compositions and calculated values using MIDS.]

\begin{tabular}{lcc}
\hline & \multicolumn{2}{c}{$\begin{array}{c}\text { \% de material em cada mistura } \\
\left(\mathrm{I}_{\mathrm{i}} / \mathrm{I}_{\text {ref }}=0,02\right)\end{array}$} \\
\cline { 2 - 3 } & Padrão & MIDS \\
\hline Caulinita & 60,0 & 58,62 \\
Ortoclásio & 25,0 & 26,2 \\
Quartzo & 0,0 & 0,0 \\
Talco & 0,0 & 9,3 \\
Acessório & 5,0 & 4,2 \\
Quartzo Livre & --- & 1,64 \\
\hline
\end{tabular}

utilizando o valor padrão das fases e o valor calculado pelo sistema MIDS, como mostrado na equação abaixo:

$$
\text { Erro }(\%)=\frac{\text { valor calculado }=\text { valor base }}{\text { valor base }}
$$

Para o cálculo do erro relativo foi utilizada a equação $\mathrm{E}$, onde se pode constatar que para os casos onde não ocorreram incompatibilidades entre as fases, o erro com relação às matérias primas simuladas é muito baixo. Com isso, pode-se classificar como satisfatórios os resultados obtidos através do método MIDS, Tabela VI.

A mistura apresentada mostrou resultado muito próximo ao padrão. Entretanto, algumas misturas apresentaram pelo menos uma fase com resultado nulo, embora estas tenham sido identificadas na análise por difração de raios X. Isso revela uma inconsistência no algoritmo. Esse problema acontece quando duas fases que estão sendo calculadas ao mesmo tempo possuem um mesmo elemento, e este tem um percentual na fase concorrente menor que $55 \%$ em relação à outra. Para que esta metodologia atenda a esses casos em particular, se faz necessário uma revisão no algoritmo dos mínimos quadrados não negativos proposto por Lawson e 
Tabela VI - Erro relativo do sistema em relação aos dados padrões e os calculados pelo método MIDS.

[Table VI - Relative error of the system with respect to standard and calculated values using MIDS.]

\begin{tabular}{lc}
\hline & Erro (\%) \\
\hline Quartzo & 0,0 \\
Caulinita & 0,023 \\
Talco & 0,07 \\
Ortoclásio & 0,05 \\
Acessório & 0,16 \\
Quartzo Livre & --- \\
Erro Médio & 0,05 \\
\hline
\end{tabular}

sua posterior correção na função pré-definida. Essa inconsistência no algoritmo torna-se crítica em regiões onde ocorram predominâncias de argilas compostas pelas fases incompatíveis, ou seja, argilas que contenham ortoclásio juntamente com ilita e/ ou moscovita, devido ao potássio $\left(\mathrm{K}_{2} \mathrm{O}\right)$; calcita juntamente com dolomita, devido ao cálcio $(\mathrm{CaO})$ e montmorilonita com clorita devido à presença do magnésio $(\mathrm{MgO})$. Nestes casos poderão haver resultados anômalos, com fases nulas em amostras onde a difração de raios X acusou a presença da mesma. Outra observação sobre os resultados obtidos pelo método MIDS foi a quantificação do quartzo livre presente na amostra que resultou na redução do valor da fase hipotética "acessórios" e, conseqüentemente, na diminuição do erro na quantificação das outras fases, já que o percentual de quartzo livre que era utilizado para o cálculo das fases presentes juntamente com o percentual total levantado pela análise química, foi isolado como uma fase independente. Anteriormente, somente após o cálculo matemático das fases presentes na amostra era considerado o que restou e acrescentado a fase "acessórios". Conseqüentemente, o quartzo livre era incluído em uma fase a qual não pertencia, alterando os percentuais das fases que possuíssem $\mathrm{SiO}_{2}$. Vale ressaltar que o tipo de curva de quartzo livre adotado, a precisão da relação da intensidade do pico da curva de quartzo da amostra em um plano pré-determinado e a intensidade do pico do padrão de quartzo adotado no mesmo plano (Ii/Iref) influencia significativamente os valores finais apresentados pelo programa. Dessa forma, é necessário o uso da curva adequada para cada análise, já que o percentual retirado da curva de referência de quartzo livre pode variar de valores muito baixos a valores consideráveis. O método MIDS permite, ainda, a resolução de sistemas sobre-determinados (mais óxidos presentes na amostra do que fases, ou mais linhas do que colunas) e sistemas subdeterminados (mais fases do que óxidos, ou mais colunas do que linhas), eliminando resultados de valores negativos, e diminuindo a quantidade de acessório através da determinação da quantidade de quartzo livre presente na amostra, que conseqüentemente são descontados da fase acessórios, onde se concentram os elementos que foram considerados excedentes.

O método apresentado para análise racional de argilominerais ainda apresenta possíveis fontes de erros, comuns a outros métodos, como a não identificação precisa de fases com a subseqüente alteração da matriz numérica, a ocorrência de fases esparsamente cristalinas e como tal não suficientemente evidenciadas na difração de raios $\mathrm{X}$, a presençade argilomineraisinterestratificadoseasimplificação da fórmula teórica de fases complexas. Todos esses fatores têm influência direta nos resultados dos cálculos e devem ser observados com muito critério para que não haja sérias discrepâncias nos resultados apresentados. Eventualmente, o método MIDS pode apresentar erros devido à presença de duas ou mais fases com a mesma fórmula teórica, como é o caso da caulinita e da haloisita e/ou a presença de grande quantidade de impurezas com elementos químicos que também estão presentes em outras fases identificadas.

\section{CONCLUSÕES}

Com base nos procedimentos de cálculos apresentados, uma rotina computacional foi desenvolvida, permitindo a quantificação mineralógica de fases de matérias-primas cerâmicas. $\mathrm{O}$ método MIDS pode ser uma ferramenta rápida $\mathrm{e}$ confiável para a determinação quantitativa de fases. Através da análise dos resultados obtidos com a utilização da metodologia proposta, verifica-se que os percentuais determinados através do algoritmo $A . X=B$ juntamente com o resultado fornecido pela curva de calibração de quartzo livre foram satisfatórios, apresentando valores bem próximos aos valores do padrão utilizado. Constatou-se, também, que a curva levantada consegue reproduzir de maneira confiável os vários percentuais de quartzo livre que possam estar presentes em uma amostra, podendo ser utilizada como ponto de referência em formulações de massa. Dessa forma, com conhecimento da matéria-prima a ser utilizada na produção da massa cerâmica, pode ser definida a necessidade de beneficiamento de uma determinada argila e ainda se este beneficiamento é viável, economizando, desta forma, tempo e investimento financeiro.

\section{REFERÊNCIAS}

[1] C. Coelho, N. Roqueiro, D. Hotza, Rational mineralogical analysis of ceramics, Mater. Lett. 52, 6 (2002) 394-398.

[2] P. Souza Santos, Ciência e Tecnologia de Argilas, S. Paulo, Editora Edgard Blücher Ltda. (1989) p. 210.

[3] B. Fabbri, C. Fiori, A. Ravaglioli, Comparation between traditional mineralogical and computarized rational analysis of ceramic raw-materials, J. Physique 47, C-1 (1986) 57-62.

[4] C. L. Lawson, R. J. Hanson, Solving least squares problems, $2^{\text {nd }}$ Ed., Philadelphia, SIAM - Society for Industrial and Applied Mathematics (1995) 337 pp.

[5] A. P. F. Albers, F. G. Melchiades, R. Machado, J. B. Baldo, A. O. Boschi, Um método simples para caracterização de argilominerais, in Anais do $45^{\circ}$ Congresso Brasileiro de Cerâmica, Santa Catarina (2001).

[6] G. P. Tomaino, Quantitative determination of quartz in calcite, dolomite and talc by powder X-ray diffraction analysis, Elsevier Science B. V., Amsterdam (1993).

(Rec. 11/03/2005, Ac. 30/05/2005) 